\title{
Topological reactions of correlation functions in partially coherent electromagnetic beams
}

\author{
Shreyas B. Raghunathan, ${ }^{1}$ Hugo F. Schouten, ${ }^{2}$ and Taco D. Visser ${ }^{1,2, *}$ \\ ${ }^{1}$ Faculty of Electrical Engineering, Mathematics and Computer Science, Delft University of Technology, Delft 2628CD, \\ The Netherlands \\ ${ }^{2}$ Department of Physics and Astronomy, and Institute for Lasers, Life and Biophotonics, VU University, Amsterdam \\ $1018 \mathrm{HV}$, The Netherlands \\ *Corresponding author: T.D.Visser@tudelft.nl
}

\author{
Received January 11, 2013; accepted February 5, 2013; \\ posted February 11, 2013 (Doc. ID 183314); published March 6, 2013
}

\begin{abstract}
It was recently shown that so-called coherence vortices, singularities of the two-point correlation function, generally occur in partially coherent electromagnetic beams. We study the three-dimensional structure of these singularities and show that in successive cross sections of a beam a rich variety of topological reactions takes place. These reactions involve, apart from vortices, the creation or annihilation of dipoles, saddles, maxima and minima of the phase of the correlation function. Since these reactions happen generically, i.e., under quite general conditions, these observations have implications for interference experiments with partially coherent, electromagnetic beams. (c) 2013 Optical Society of America

OCIS codes: $\quad 030.1640,050.1940,260.1960,260.2110,260.6042$.
\end{abstract}

\section{INTRODUCTION}

It is well known that wave fields exhibit remarkable structures near their zeros of intensity [1] ]. These zeros may exist briefly, for example, when pulsed fields are interfering with each other, or they may be permanent, when the fields are monochromatic. At such a zero, the amplitude of the field vanishes and its phase is, therefore, undefined or singular. Around these phase singularities the phase typically has a vortex-like behavior. Apart from phase singularities, phase dipoles, phase extrema, and phase saddles may also occur. These different structures can exist arbitrarily close to one another, and can in fact be created or annihilated in so-called topological reactions, see for example [2-7].

The most often-studied phase singularities are those of scalar wave fields, the Airy rings of focal fields being a prime example, see [8, Section 8.8.4] and [9]. Singularities of the Poynting vector have also been analyzed. These occur in Sommerfeld's diffraction problem [8, Section 11.5], in focused fields [10], and in the transmission of light by subwavelength apertures $[\underline{11}, \underline{12}]$. Singularities of individual Cartesian components of the electric field vector have also been described in focal fields [13]. Studies of this type (and also of polarization singularities, with which we will not be concerned here) have given rise to the relatively new discipline of singular optics. Reviews are presented in $[\underline{14}, \underline{15}]$.

In recent years, singular optics has been expanded to include partially coherent wave fields. Many fields that are encountered in practice, such as those generated by multimode lasers or fields that have traveled through atmospheric turbulence, belong to this category. In such fields the phase is a random quantity and therefore they do not contain "traditional" phase singularities. However, the statistical properties of these fields are described by two-point correlation functions, which do have a definite phase [16-18]. A few years ago it was pointed out that these functions can also exhibit singular behavior [19]. Such correlation singularities, or "coherence vortices," occur at pairs of points at which the field is completely uncorrelated. Coherence vortices have since been found in optical beams [20-27], in focused fields [28], in the far-zone of quasi-homogeneous sources [29], and in fields produced by Mie scattering [30,31]. Some of these studies have been carried out in the space-time domain, others in the space-frequency domain. Here we will use the latter approach. This means that the main two-point correlation function we will be dealing with is the spectral degree of coherence [16]. Just like their monochromatic counterparts, coherence vortices can also undergo topological reactions. Thus far such reactions have hardly been studied. Notable exceptions are [32-34].

In all the coherence studies mentioned above, the analysis was limited to scalar wave fields. Only recently has it has been shown that coherence singularities occur generically in partially coherent electromagnetic beams [35]. For the wide class of electromagnetic Gaussian Schell-model (GSM) beams [17] it was demonstrated that, even in the absence of ordinary phase singularities, the spectral degree of coherence typically displays singular behavior. (Notice that scalar GSM beams have no such coherence singularities.)

In this article we demonstrate that electromagnetic GSM beams are intrinsically three-dimensional in nature. We illustrate this by examining the structure of surfaces of equal phase of the correlation function. In particular, this threedimensional character implies that different beam cross sections have different topological features [2]. Thus, an observer moving through successive cross-sectional planes will notice a sequence of topological reactions. From the conservation of topological charge and topological index [14,36], it is to be expected that the creation or annihilation of coherence vortices 
involves phase saddles. We find this to be the case, but reactions between phase extrema (maxima and minima), dipoles, and phase saddles of the correlation function are also observed. As we will show, a rich variety of topological reactions occurs on propagation of these partially coherent, electromagnetic GSM beams. The observation that different cross sections of a GSM beam have different coherence properties, has profound implications for their use in scattering [37] and interference experiments [17].

\section{PARTIALLY COHERENT ELECTROMAGNETIC BEAMS}

The properties of partially coherent electromagnetic beams are described in detail in a textbook by Wolf [17]. Here, we summarize some of the main definitions. The state of coherence and polarization of a random beam that propagates along the $z$-axis is characterized by its electric cross-spectral density matrix

$$
\mathbf{W}\left(\mathbf{r}_{1}, \mathbf{r}_{2}, \omega\right)=\left(\begin{array}{ll}
W_{x x}\left(\mathbf{r}_{1}, \mathbf{r}_{2}, \omega\right) & W_{x y}\left(\mathbf{r}_{1}, \mathbf{r}_{2}, \omega\right) \\
W_{y x}\left(\mathbf{r}_{1}, \mathbf{r}_{2}, \omega\right) & W_{y y}\left(\mathbf{r}_{1}, \mathbf{r}_{2}, \omega\right)
\end{array}\right),
$$

where

$$
W_{i j}\left(\mathbf{r}_{1}, \mathbf{r}_{2}, \omega\right)=\left\langle E_{i}^{*}\left(\mathbf{r}_{1}, \omega\right) E_{j}\left(\mathbf{r}_{2}, \omega\right)\right\rangle, \quad(i, j=x, y)
$$

Here, $E_{i}(\mathbf{r}, \omega)$ is a Cartesian component of the electric field at a point $\mathbf{r}$ at frequency $\omega$, of a typical realization of the statistical ensemble representing the beam, and the angled brackets indicate the ensemble average. The spectral degree of coherence $\eta\left(\mathbf{r}_{1}, \mathbf{r}_{2}, \omega\right)$ of the field is defined as

$$
\eta\left(\mathbf{r}_{1}, \mathbf{r}_{2}, \omega\right)=\frac{\operatorname{Tr} \mathbf{W}\left(\mathbf{r}_{1}, \mathbf{r}_{2}, \omega\right)}{\left[\operatorname{Tr} \mathbf{W}\left(\mathbf{r}_{1}, \mathbf{r}_{1}, \omega\right) \operatorname{Tr} \mathbf{W}\left(\mathbf{r}_{2}, \mathbf{r}_{2}, \omega\right)\right]^{1 / 2}},
$$

where Tr denotes the trace. A correlation singularity occurs at pairs of points for which $\eta\left(\mathbf{r}_{1}, \mathbf{r}_{2}, \omega\right)=0$. (From now on we suppress the $\omega$-dependence of the various quantities.)

The presence of correlation singularities in a wave field has several consequences. First, when the fields at points $\mathbf{r}_{1}$ and $\mathbf{r}_{2}$ are combined in Young's experiment, the visibility of the ensuing interference fringes crucially depends on the value of $\eta\left(\mathbf{r}_{1}, \mathbf{r}_{2}\right)$, see [17, Section 9.2]. At a singularity, where $\eta\left(\mathbf{r}_{1}, \mathbf{r}_{2}\right)=0$, the fringe visibility will be zero. This is because the local modulations of $\left|E_{x}\right|^{2}$ and $\left|E_{y}\right|^{2}$ on the observation screen have equal magnitude and opposite sign, resulting in a zero visibility of the total spectral density. Second, in experiments of the Hanbury Brown-Twiss type one determines the correlation of intensity fluctuations at two points [38]. These higher-order correlations depend on the so-called degree of cross polarization [39]. Correlation singularities coincide with a divergence of the degree of cross polarization, the consequences of which are discussed in [40]. Furthermore, it is to be noted that the phase singularities found in monochromatic fields and the coherence singularities of partially coherent fields are not independent of one another. The former can evolve into the latter when the coherence of the field

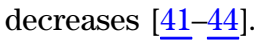

Since we are dealing with beams, it is natural to investigate the possible occurence of coherence vortices in a transverse plane $z=$ constant (see Fig. 1). We therefore set $\mathbf{r}_{1}=\left(\rho_{1}, z\right)$

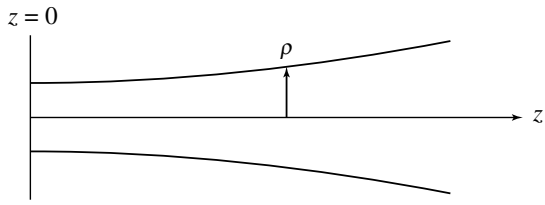

Fig. 1. Illustrating the notation. A partially coherent, electromagnetic GSM beam propagates in the $z$-direction. The source plane is taken to be at $z=0$. The vector $\rho=(x, y)$ indicates a transverse position.

and $\mathbf{r}_{2}=\left(\rho_{2}, \boldsymbol{z}\right)$. According to Eq. (י) , a coherence vortex exists when both

$$
\left|W_{x x}\left(\rho_{1}, \rho_{2}, z\right)\right|=\left|W_{y y}\left(\rho_{1}, \rho_{2}, z\right)\right|,
$$

and

$$
\arg \left[W_{x x}\left(\rho_{1}, \rho_{2}, z\right)\right]-\arg \left[W_{y y}\left(\rho_{1}, \rho_{2}, z\right)\right]=\pi(\bmod 2 \pi),
$$

where arg denotes the argument or phase of the matrix element.

\section{ELECTROMAGNETIC GSM BEAMS}

GSM beams [17] form a wide class of partially coherent electromagnetic beams that includes the lowest-order Gaussian laser mode. For such beams the elements of the cross-spectral density matrix in the source plane $z=0$ read

$W_{i j}\left(\rho_{1}, \rho_{2}, z=0\right)=\sqrt{S_{i}\left(\rho_{1}\right) S_{j}\left(\rho_{2}\right)} \mu_{i j}\left(\rho_{2}-\rho_{1}\right), \quad(i, j=x, y)$

with the spectral densities of the two individual components of the electric field vector $S_{i}(\rho)=W_{i i}(\rho, \rho)$ and the correlation coefficient $\mu_{i j}\left(\rho_{2}-\rho_{1}\right)$ both assumed to be Gaussian functions, i.e.,

$$
\begin{gathered}
S_{i}(\boldsymbol{\rho})=A_{i}^{2} \exp \left(-\boldsymbol{\rho}^{2} / 2 \sigma_{i}^{2}\right) \\
\mu_{i j}\left(\rho_{2}-\rho_{1}\right)=B_{i j} \exp \left[-\left(\rho_{2}-\rho_{1}\right)^{2} / 2 \delta_{i j}^{2}\right]
\end{gathered}
$$

The parameters $A_{i}, B_{i j}, \sigma_{i}$, and $\delta_{i j}$ are independent of position, but may depend on the frequency $\omega$. In addition, they have to satisfy certain constraints to ensure that the field is beam-like [17]. As the beam propagates to a plane $z>0$, and if we take $\overline{\sigma_{x}}=\sigma_{y}=\sigma$, the matrix elements become (see [17], where the one but last minus sign of Eq. (10) on p. 184 should be a plus sign)

$$
\begin{aligned}
W_{i j}\left(\boldsymbol{\rho}_{1}, \boldsymbol{\rho}_{2}, \boldsymbol{z}\right)= & \frac{A_{i} A_{j} B_{i j}}{\Delta_{i j}^{2}(\boldsymbol{z})} \exp \left[-\frac{\left(\boldsymbol{\rho}_{1}+\boldsymbol{\rho}_{2}\right)^{2}}{8 \sigma^{2} \Delta_{i j}^{2}(z)}\right] \\
& \times \exp \left[-\frac{\left(\boldsymbol{\rho}_{2}-\boldsymbol{\rho}_{1}\right)^{2}}{2 \Omega_{i j}^{2} \Delta_{i j}^{2}(z)}\right] \exp \left[\frac{i k\left(\boldsymbol{\rho}_{2}^{2}-\boldsymbol{\rho}_{1}^{2}\right)}{2 R_{i j}(\boldsymbol{z})}\right],
\end{aligned}
$$

where

$$
\Delta_{i j}^{2}(z)=1+\left(z / k \sigma \Omega_{i j}\right)^{2}
$$




$$
\begin{gathered}
\frac{1}{\Omega_{i j}^{2}}=\frac{1}{4 \sigma^{2}}+\frac{1}{\delta_{i j}^{2}}, \\
R_{i j}(z)=\left[1+\left(k \sigma \Omega_{i j} / z\right)^{2}\right] z
\end{gathered}
$$

with $k=\omega / c$ the wavenumber associated with frequency $\omega$, $c$ being the speed of light in vacuum.

We note that the diagonal matrix elements as given by Eq. (6) are real-valued and positive. Therefore, according to Eq. (5), there are no correlation singularities in the source plane. However, as we will illustrate in Section 4 , such singularities can be created as the beam propagates.

\section{THREE-DIMENSIONAL STRUCTURE OF CORRELATION SINGULARITIES}

Let us first analyze the shape of the surfaces of equal phase of the correlation function when there are no coherence singularities present. We choose a fixed point of reference $\rho_{1}$ and then calculate the phase of $\eta\left(\rho_{1}, \rho_{2}, z\right)$. An example for $\arg \eta\left(\rho_{1}, \rho_{2}, z\right)=\pi$ is shown in Fig. 2 , where two sheets of this constant phase can be seen. (On increasing the field of view, more of these sheets become visible.) If we slightly reduce the value of one of the correlation lengths to $\delta_{y y}=0.14 \mathrm{~mm}$, the two initially smooth surfaces get somewhat "dented," as is shown in Fig. 3. These dents correspond to minima of the phase in transverse cross sections of the beam (i.e., planes for which $z=$ constant), and will be discussed in Section 5 .

On further decreasing $\delta_{y y}$ to $0.12 \mathrm{~mm}$, correlation singularities come into existence. These lines of coherence vortices form a closed string, as shown in Fig. 4 . For all values of the phase, surfaces of equal phase end on this string. This can either happen from "within" the string, or from "outside" of the string. The former leads to a protrusion of the phase surface, the latter leads to a hole in the surface. As is seen from

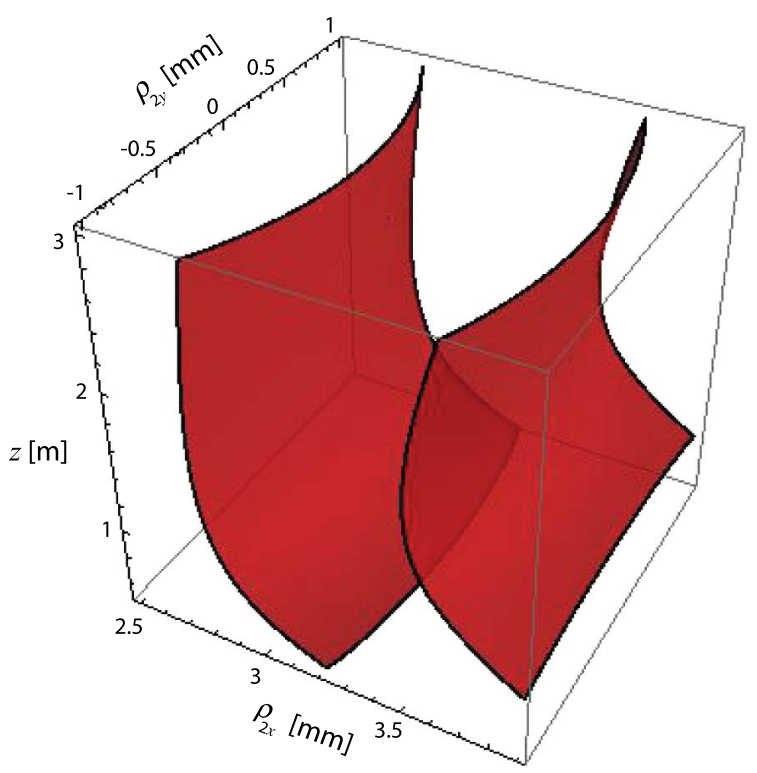

Fig. 2. (Color online) Two surfaces for which the phase of $\eta\left(\rho_{1}, \rho_{2}, z\right)$ equals $\pi$. In this case $\delta_{y y}=0.18 \mathrm{~mm}$. The other parameters are $\lambda=632.8 \mathrm{~nm}, \delta_{x x}=0.2 \mathrm{~mm}, \sigma=1 \mathrm{~mm}, A_{x}=1$, and $A_{y}=3$. The reference point $\rho_{1}=(2.5,0) \mathrm{mm}$ (Media 1$)$.

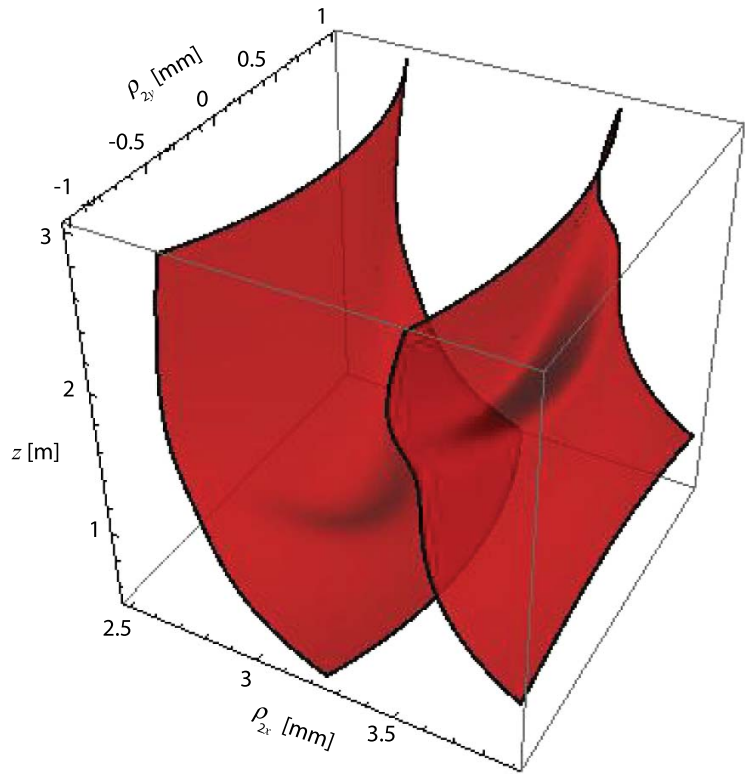

Fig. 3. (Color online) Two surfaces for which the phase of $\eta\left(\rho_{1}, \rho_{2}, z\right)$ equals $\pi$. In this case $\delta_{y y}=0.14 \mathrm{~mm}$.

Fig. 5, both cases happen simultaneously: the string (indicated in green) borders both a hole and a protrusion of the phase sheet. Notice that the left-hand sheet is now dented even more. If we further decrease the value of $\delta_{y y}$, the string of coherence vortices increases in size, and extends to both surfaces of equal phase, as is shown in Fig. 6. The protrusion of Fig. $\underline{5}$ has grown in size and now connects the two sheets. When the value of $\delta_{y y}$ decreased even more, the string of singularities gradually moves to the left (i.e., to smaller values of $\rho_{2 x}$ ), and only intersects the left-hand phase sheet. The right-hand sheet has returned to its previous smooth state. This is shown in Fig. 7.

From the complicated three-dimensional nature of the correlation function, as illustrated in Figs. 2-2, it follows that different transverse cross sections of the beam will have quite different topological features. Therefore, an observer moving

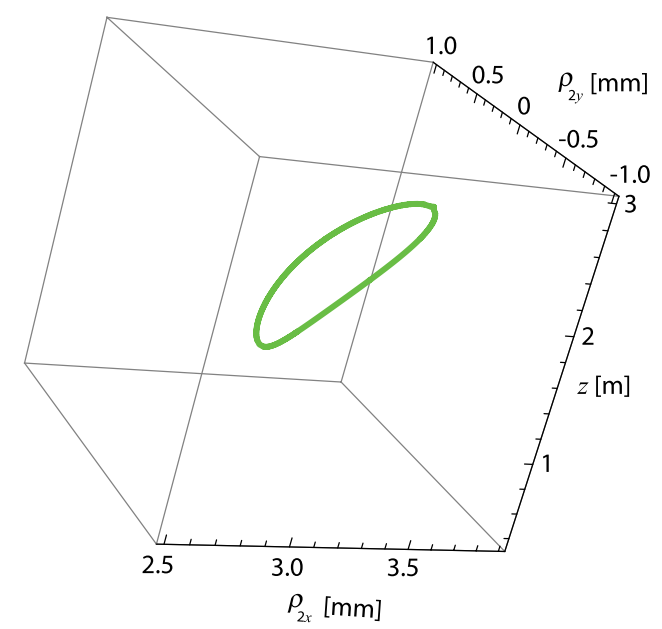

Fig. 4. (Color online) Closed string of coherence singularities (green curve). 


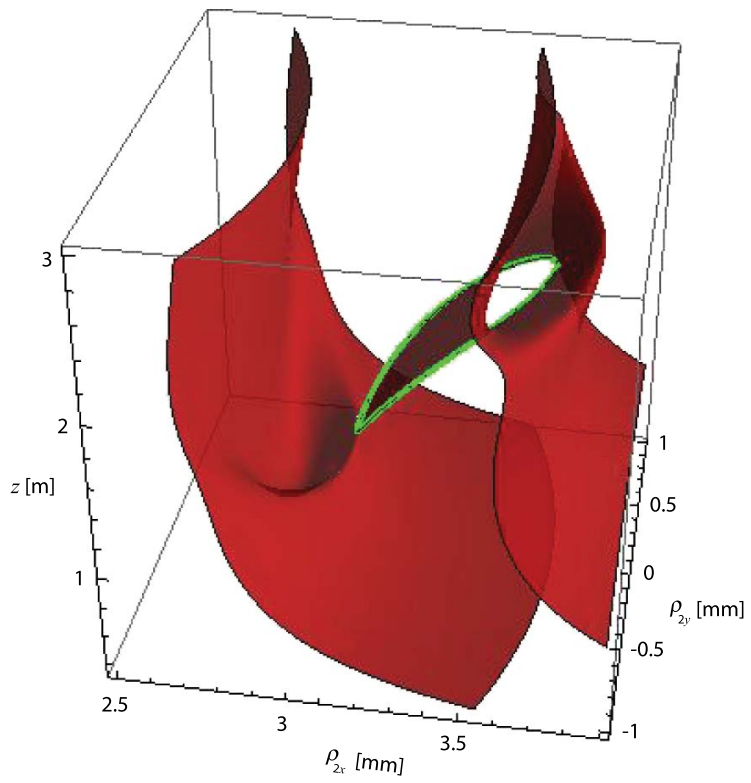

Fig. 5. (Color online) Two surfaces for which the phase of $\eta\left(\rho_{1}, \rho_{2}, z\right)$ equals $\pi$. In this case $\delta_{y y}=0.12 \mathrm{~mm}$. A closed string of coherence vortices (green curve) has come into existence. The right-hand phase sheet terminates on the string, creating a hole and a protrusion of the sheet.

from one transverse plane to another, witnesses a series of topological reactions, as will be discussed in the next section.

\section{TOPOLOGICAL REACTIONS}

As noted above, the phase of monochromatic fields typically has a vortex-like behavior around a phase singularity. This is also true for the phase of the spectral degree of coherence $\eta\left(\rho_{1}, \rho_{2}, z\right)$ around a correlation singularity. On keeping $\rho_{1}$

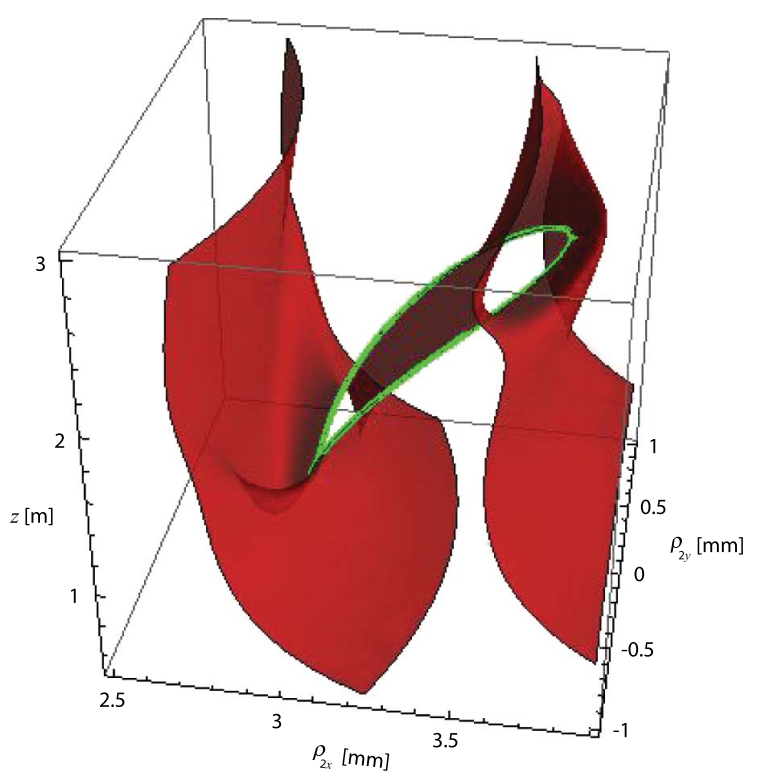

Fig. 6. (Color online) Single surface for which the phase of $\eta\left(\rho_{1}, \rho_{2}, z\right)$ equals $\pi$. In this case $\delta_{y y}=0.11 \mathrm{~mm}$. The string of coherence vortices (green curve) has expanded, causing the protrusion of Fig. 5 to grow. The two formerly disjointed phase sheets are now connected.

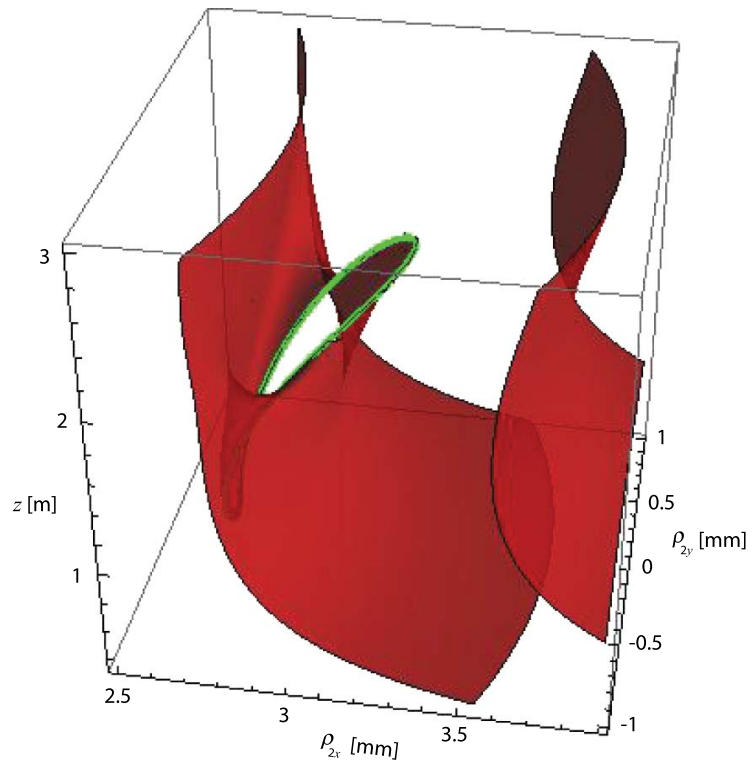

Fig. 7. (Color online) Two surfaces for which the phase of $\eta\left(\rho_{1}, \rho_{2}, z\right)$ equals $\pi$. In this case $\delta_{y y}=0.06 \mathrm{~mm}$. The string of coherence vortices (green curve) has moved sideways and now only intersects the lefthand phase sheets. The two phase sheets are again disconnected.

fixed, while traversing in a counterclockwise manner, a closed circuit in the $\rho_{2}, z$-plane which encompasses a single singularity, the phase of $\eta\left(\rho_{1}, \rho_{2}, z\right)$ changes by an amount of $2 n \pi$. The nonzero integer $n$ is called the topological charge. To the singularities (vortices and dipoles) and to the stationary points (extrema and saddles) of the phase of the correlation function we can also assign a topological index [45], which is defined as the topological charge of the phase singularities of the vector field $\nabla_{\perp} \arg \left[\eta\left(\rho_{1}, \rho_{2}, \boldsymbol{z}\right)\right]$, where $\nabla_{\perp}$ denotes differentiation with respect to $\rho_{2}$. In topological reactions both the charge and index are conserved quantities [36]. In Table 1, they are listed for different types of points.

In the following examples we first choose a fixed reference point $\rho_{1}$, and then, keeping all other parameters fixed, we calculate the phase contours of $\eta\left(\rho_{1}, \rho_{2}, z\right)$ in successive cross sections of the beam. (Changing the choice of the reference point may lead to different topological reactions, as was remarked by Gu and Gbur [33].) A first result is shown in Fig. 8 for the plane $z=0.1 \mathrm{~m}$. On the left there is a phase minimum together with a phase saddle (the intersection of the red contour line with itself), whereas on the right a phase maximum and another phase saddle can be seen. If the plane of observation is moved away from the source, the minimum and the nearby saddle gradually move together and the phase of $\eta\left(\rho_{1}, \rho_{2}, z\right)$ at the minimum and at the saddle point converge. This goes on until the minimum and the saddle annihilate each

Table 1. Topological Charge and Index of Singular and Stationary Points

\begin{tabular}{lcc}
\hline & Charge & Index \\
\hline Vortex & \pm 1 & 1 \\
Saddle & 0 & -1 \\
Maximum & 0 & 1 \\
Minimum & 0 & 1 \\
Dipole & 0 & 2 \\
\hline
\end{tabular}




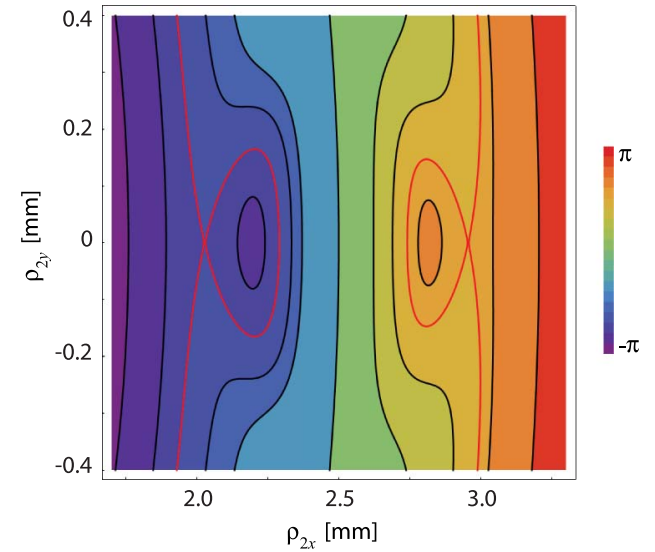

Fig. 8. (Color online) Phase contours of $\eta\left(\rho_{1}, \rho_{2}, z\right)$ in the plane $z=0.1 \mathrm{~m}$. A minimum, a maximum, and two saddle points (intersections of the red curves) can be seen. In this and in the following examples we have taken $\rho_{1}=(2.5,0) \mathrm{mm}, A_{x}=1, A_{y}=3, \sigma=1 \mathrm{~mm}$, $\delta_{x x}=0.2 \mathrm{~mm}, \delta_{y y}=0.12 \mathrm{~mm}$, and the wavelength $\lambda=633 \mathrm{~nm}$.

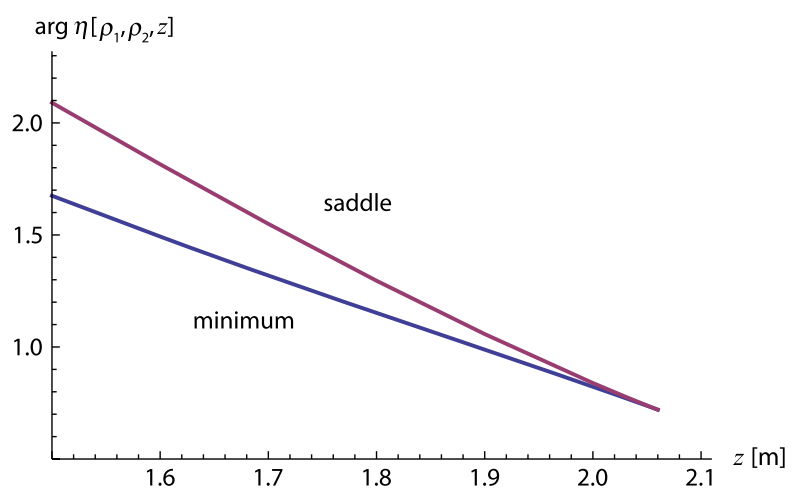

Fig. 9. (Color online) Phase of $\eta\left(\boldsymbol{\rho}_{1}, \boldsymbol{\rho}_{2}, \boldsymbol{z}\right)$ at the minimum and at the phase saddle (visible on the left-hand side in Fig. 8), in various cross sections of the beam.

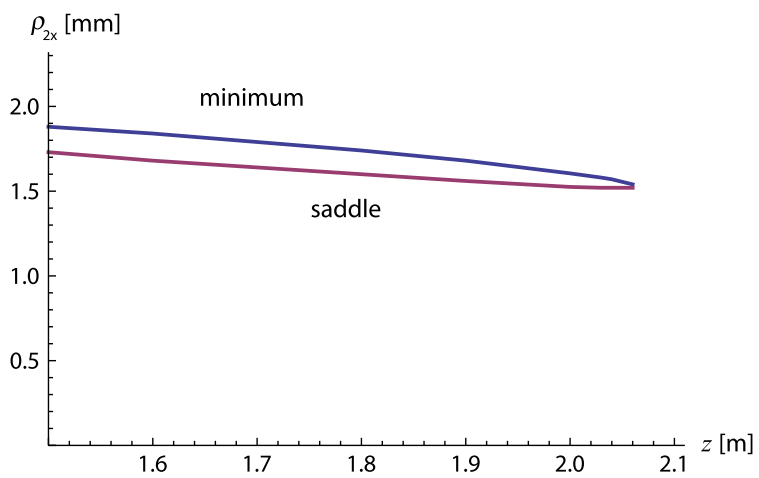

Fig. 10. (Color online) Position $\rho_{2 x}$ of the minimum (blue curve) and that of the saddle (red curve) in various cross sections of the beam. Near $z=2.06 \mathrm{~m}$ the minimum and the saddle point annihilate each other.

other near $z=2.06 \mathrm{~m}$. This process is illustrated in Figs. $\underline{9}$ and 10.

From here on we will concentrate on the maximum and its nearby saddle point of Fig. $\underline{8}$ which, as we will see, go through a rich series of topological reactions in which both the zero total topological charge and the zero topological index are

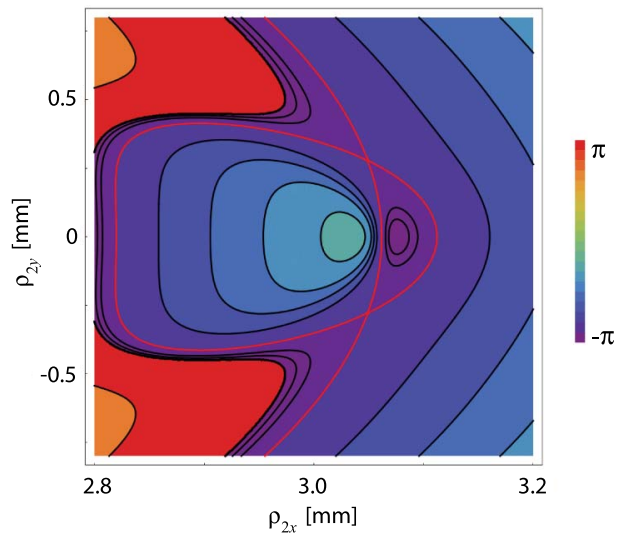

Fig. 11. (Color online) Phase contours of $\eta\left(\boldsymbol{\rho}_{1}, \boldsymbol{\rho}_{2}, \boldsymbol{z}\right)$ in the plane $z=1.12 \mathrm{~m}$. The right-hand side phase saddle of Fig. $\underline{8}$ has decayed into a minimum and two saddles (intersections of the two red curves).

conserved. As the plane of observation is gradually moved away from the source, the saddle decays into a minimum and two saddle points (near $z=0.89 \mathrm{~m}$ ). The end result of this reaction is shown in Fig. 11.

On further moving the cross-sectional plane another reaction occurs: the two phase extrema move closer to each other (along the $\rho_{2 x}$-axis) until they form a dipole [46] with index 2 (near $z=1.18 \mathrm{~m}$ ), as is illustrated in Fig. 12 . A dipole is formed when the cross-sectional plane is tangential to the vortex string shown in Fig. $\underline{4}$. This dipole immediately decays into two phase vortices with opposite topological charge, which in successive cross sections gradually move away from each other along the $\rho_{2 y}$-direction. The result is depicted in Fig. 13. Notice that two vortices occur whenever the cross-sectional plane intersects the vortex string at two points.

According to Eq. (12), the factor $R_{i j}(z)$ becomes infinite as $z \rightarrow \infty$. This implies that in that limit Eq. (5) can no longer be satisfied, since both diagonal elements of the cross-spectral density matrix become real-valued and positive. Therefore, the correlation vortices must eventually disappear. Indeed we find that near $z=2.35 \mathrm{~m}$ the two vortices briefly form a second dipole, which decays into a maximum and a minimum. These two phase extrema, together with the two remaining saddle points, are shown in Fig. 14.

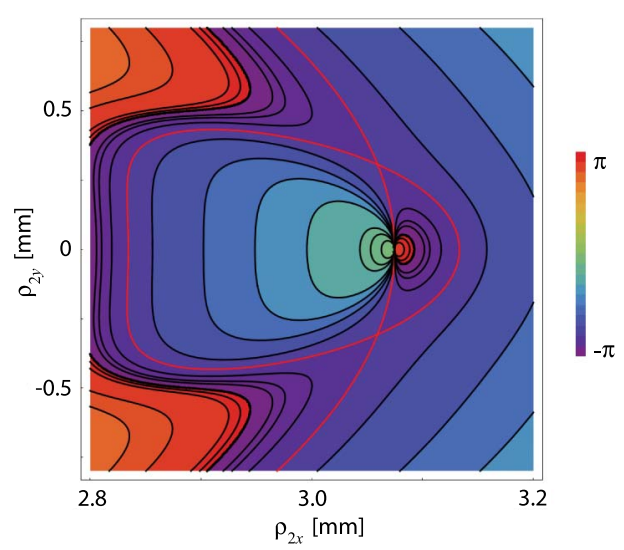

Fig. 12. (Color online) Phase contours of $\eta\left(\rho_{1}, \rho_{2}, z\right)$ in the plane $z=1.1808 \mathrm{~m}$, containing a dipole and two saddle points (intersections of the red curves). 


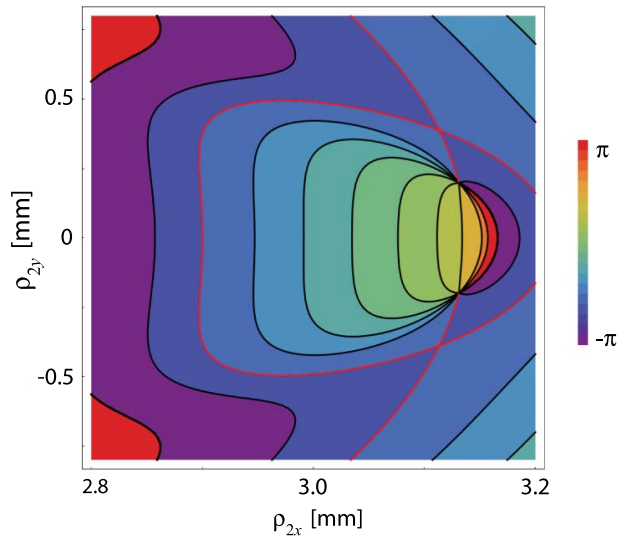

Fig. 13. (Color online) Phase contours of $\eta\left(\rho_{1}, \boldsymbol{\rho}_{2}, \boldsymbol{z}\right)$ in the plane $z=1.4 \mathrm{~m}$, containing two vortices ("coherence singularities") and two saddle points (intersections of the red curves).

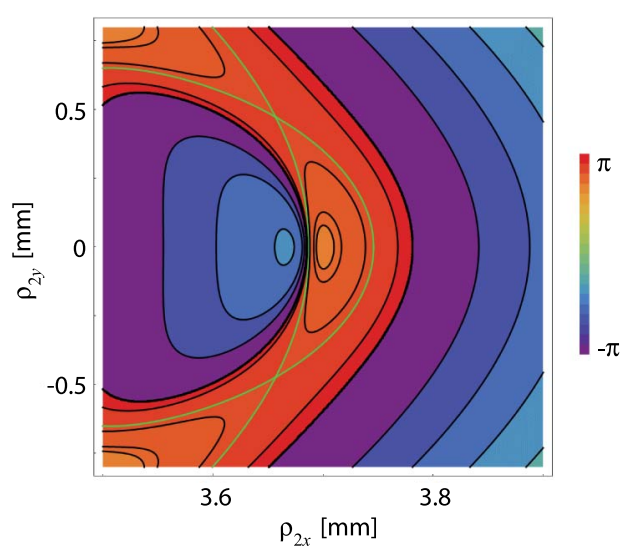

Fig. 14. (Color online) Phase contours of $\eta\left(\rho_{1}, \rho_{2}, z\right)$ in the plane $z=2.4 \mathrm{~m}$, containing a maximum, a minimum, and two saddle points (intersections of the green curves).

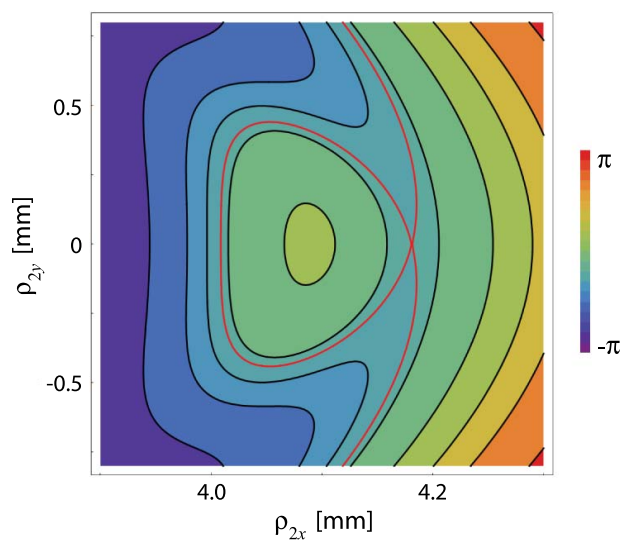

Fig. 15. (Color online) Phase contours of $\eta\left(\rho_{1}, \rho_{2}, z\right)$ in the plane $z=3.0 \mathrm{~m}$, containing a maximum and a saddle (intersection of the red curve).

The next reaction takes place in the plane $z=2.81 \mathrm{~m}$. There the minimum and the two saddle points merge together to form a single saddle point. This is illustrated in Fig. 15.

The final reaction occurs at $z=3.33 \mathrm{~m}$. The maximum and the saddle annihilate each other, leaving a field without topological features, as is depicted in Fig. 16.

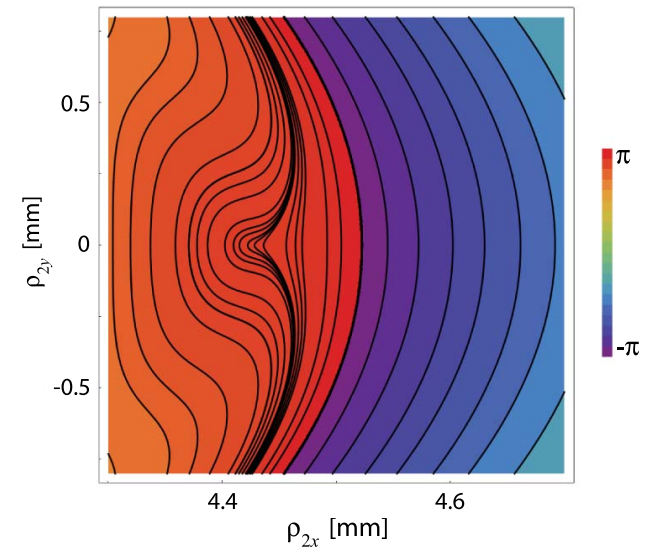

Fig. 16. (Color online) Phase contours of $\eta\left(\boldsymbol{\rho}_{1}, \boldsymbol{\rho}_{2}, \boldsymbol{z}\right)$ in the plane $z=3.33 \mathrm{~m}$, right after the final topological reaction. There are no more singularities or stationary points.

\section{CONCLUSIONS}

We have studied the properties of the correlation function of an electromagnetic partially coherent beam of the GSM class. Although the spectral density of such beams has no singular points, the phase of its correlation function does show a rich variety of saddles, extrema, dipoles, and vortices. The structure of the correlation function is found to be essentially three-dimensional. This was illustrated by its complex-shaped surfaces of equal phase. On smoothly changing a parameter that characterizes the beam, these surfaces are first slightly deformed and then torn when correlation singularities come into existence. Since different cross sections of the beam have different topological features, an observer moving from one transverse plane to another, will witnesses a series of complicated topological reactions. In all these reactions the topological charge and the topological index are conserved. We emphasize that all these reactions are generic, i.e., they occur quite generally and not just for special choices of the parameters that characterize the beam. The observation that different cross sections of partially coherent electromagnetic beams have quite different coherence properties has profound implications for interference and scattering experiments with such beams.

\section{ACKNOWLEDGMENT}

T. D. Visser's research is supported by the Plasmonics program of the Foundation for Fundamental Research on Matter (FOM).

\section{REFERENCES}

1. J. F. Nye and M. V. Berry, "Dislocations in wave trains," Proc. R. Soc. A 336, 165-190 (1974).

2. I. Freund, "Critical foliations," Opt. Lett. 26, 545-547 (2001).

3. I. Freund, "Optical vortex trajectories," Opt. Commun. 181, 19-33 (2000).

4. I. Freund and D. A. Kessler, "Critical point trajectory bundles in singular wave fields,” Opt. Commun. 187, 71-90 (2001).

5. G. Molina-Terriza, J. Recolons, J. P. Torres, L. Torner, and E. M. Wright, "Observation of the dynamical inversion of the topological charge of an optical vortex,” Phys. Rev. Lett. 87, 023902 (2001).

6. A. Ya. Bekshaev, M. S. Soskin, and M. V. Vasnetsov, "Transformation of higher-order optical vortices upon focusing by an astigmatic lens,” Opt. Commun. 241, 237-247 (2004). 
7. A. Bezryadina, D. N. Neshev, A. S. Desyatnikov, J. Young, Z. Chen, and Y. S. Kivshar, "Observation of topological transformations of optical vortices in two-dimensional photonic lattices," Opt. Express 14, 8317-8327 (2006).

8. M. Born and E. Wolf, Principles of Optics: Electromagnetic Theory of Propagation, Interference and Diffraction of Light, 7th (expanded) ed. (Cambridge University, 1999).

9. G. P. Karman, A. van Duijl, and J. P. Woerdman, "Creation and annihilation of phase singularities in a focal field," Opt. Lett. 22, 1503-1505 (1997)

10. A. Boivin, J. Dow, and E. Wolf, "Energy flow in the neighborhood of the focus of a coherent beam," J. Opt. Soc. Am. 57, 1171-1175 (1967).

11. H. F. Schouten, G. Gbur, T. D. Visser, D. Lenstra, and H. Blok, "Creation and annihilation of phase singularities near a subwavelength slit," Opt. Express 11, 371-380 (2003).

12. H. F. Schouten, T. D. Visser, and D. Lenstra, "Optical vortices near sub-wavelength structures,” J. Opt. B 6, S404-S409 (2004).

13. D. W. Diehl and T. D. Visser, "Phase singularities of the longitudinal field components in high-aperture systems," J. Opt. Soc. Am. A 21, 2103-2108 (2004).

14. J. F. Nye, Natural Focusing and Fine Structure of Light (IOP Publishing, 1999).

15. M. S. Soskin and M. V. Vasnetsov, "Singular optics," in Progress in Optics, E. Wolf, ed. (Elsevier, 2001), Vol. 42, pp. 83-110.

16. L. Mandel and E. Wolf, Optical Coherence and Quantum Optics (Cambridge University, 1995)

17. E. Wolf, Introduction to the Theory of Coherence and Polarization of Light (Cambridge University, 2007).

18. G. Gbur and T. D. Visser, "The structure of partially coherent fields," in Progress in Optics, E. Wolf, ed. (Elsevier, 2010), Vol. 55, pp. 285-341.

19. H. F. Schouten, G. Gbur, T. D. Visser, and E. Wolf, "Phase singularities of the coherence functions in Young's interference pattern," Opt. Lett. 28, 968-970 (2003).

20. G. V. Bogatyryova, C. V. Fel'de, P. V. Polyanskii, S. A. Ponomarenko, M. S. Soskin, and E. Wolf, "Partially coherent vortex beams with a separable phase," Opt. Lett. 28, 878-880 (2003).

21. G. Gbur and T. D. Visser, "Coherence vortices in partially coherent beams," Opt. Commun. 222, 117-125 (2003).

22. D. M. Palacios, I. D. Maleev, A. S. Marathay, and G. A. Swartzlander, Jr., "Spatial correlation singularity of a vortex field," Phys. Rev. Lett. 92, 143905 (2004).

23. G. A. Swartzlander, Jr. and J. Schmit, "Temporal correlation vortices and topological dispersion," Phys. Rev. Lett. 93, 093901 (2004).

24. I. D. Maleev, D. M. Palacios, A. S. Marathay, and G. A Swartzlander, Jr., "Spatial correlation vortices in partially coherent light: theory," J. Opt. Soc. Am. A 21, 1895-1900 (2004).

25. W. Wang, Z. Duan, S. G. Hanson, Y. Miyamoto, and M. Takeda, "Experimental study of coherence vortices: local properties of phase singularities in a spatial coherence function," Phys. Rev. Lett. 96, 073902 (2006).

26. G. A. Swartzlander, Jr. and R. I. Hernandez-Aranda, "Optical Rankine vortex and anomalous circulation of light," Phys. Rev. Lett. 99, 163901 (2007).
27. T. van Dijk and T. D. Visser, "Evolution of singularities in a partially coherent vortex beam," J. Opt. Soc. Am. A 26, 741-744 (2009).

28. D. G. Fischer and T. D. Visser, "Spatial correlation properties of focused partially coherent light," J. Opt. Soc. Am. A 21 , 2097-2102 (2004).

29. T. van Dijk, H. F. Schouten, and T. D. Visser, "Coherence singularities in the field generated by partially coherent sources," Phys. Rev. A 79, 033805 (2009).

30. M. L. Marasinghe, M. Premaratne, and D. M. Paganin, "Coherence vortices in Mie scattering of statistically stationary partially coherent fields," Opt. Express 18, 6628-6641 (2010).

31. M. L. Marasinghe, M. Premaratne, D. M. Paganin, and M. A. Alonso, "Coherence vortices in Mie scattered nonparaxial partially coherent beams," Opt. Express 20, 2858-2875 (2012)

32. W. Wang and M. Takeda, "Coherence current, coherence vortex, and the conservation law of coherence," Phys. Rev. Lett. 96, 223904 (2006).

33. Y. Gu and G. Gbur, "Topological reactions of optical correlation vortices," Opt. Commun. 282, 709-716 (2009).

34. M. L. Marasinghe, D. M. Paganin, and M. Premaratne, "Coherence-vortex lattice formed via Mie scattering of partially coherent light by several dielectric nanospheres," Opt. Lett. 36, 936-938 (2011).

35. S. B. Raghunathan, H. F. Schouten, and T. D. Visser, "Correlation singularities in partially coherent electromagnetic beams," Opt. Lett. 37, 4179-4181 (2012).

36. S. H. Strogatz, Nonlinear Dynamics and Chaos (AddisonWesley, 1994), pp. 174-180.

37. T. van Dijk, D. G. Fischer, T. D. Visser, and E. Wolf, "Effects of spatial coherence on the angular distribution of radiant intensity generated by scattering on a sphere," Phys. Rev. Lett. 104, 173902 (2010).

38. R. Hanbury Brown and R. Q. Twiss, "Correlation between photons in two coherent beams of light," Nature 177, 27-29 (1956).

39. S. N. Volkov, D. F. V. James, T. Shirai, and E. Wolf, "Intensity fluctuations and the degree of cross-polarization in stochastic electromagnetic beams," J. Opt. A 10, 055001 (2008).

40. T. Hassinen, J. Tervo, T. Setälä, and A. T. Friberg, "Hanbury Brown-Twiss effect with electromagnetic waves," Opt. Express 19, 15188-15195 (2011).

41. G. Gbur and G. A. Swartzlander, Jr., "Complete transverse representation of a correlation singularity of a partially coherent field," J. Opt. Soc. Am. B 25, 1422-1429 (2008).

42. G. Gbur, T. D. Visser, and E. Wolf, "Hidden singularities in partially coherent wavefields," J. Opt. A 6, S239-S242 (2004).

43. G. Gbur and T. D. Visser, "Phase singularities and coherence vortices in linear optical systems," Opt. Commun. 259, 428-435 (2006).

44. T. D. Visser and R. W. Schoonover, "A cascade of singular field patterns in Young's interference experiment," Opt. Commun. 281, 1-6 (2008).

45. J. F. Nye, "Unfolding of higher-order wave dislocations," J. Opt. Soc. Am. A 15, 1132-1138 (1998).

46. C. Hsiung, A First Course in Differential Geometry (International, 1997), p. 266 